\title{
Exposé introductif sur les crues de la Cèze, des Gardons et du Vidourle des 30 septembre et 4 octobre 1958
}

\author{
Introductory discussion on the floods \\ of the Cèze, Gardons and Vidourle \\ on September 30th and October 4th 1958
}

PAR Ph. DEYMIÉ

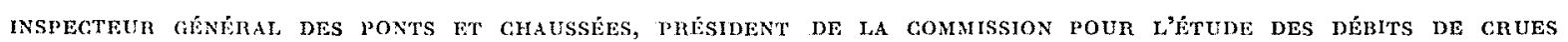

FRÉQUENCE DES CRUES

Nous avons relevé dans une intéressante note de M. Schenk, publiée par le Bulletin de la Société d'études météorologiques et climatologiques du Gard et des Cévennes, les dates des grandes crues qui ont laissé un souvenir à Alès (Gardon d'Alès) :

- 21 septembre 1471 (plusieurs morts);

- Septembre 1551;

- 10 septembre 1604 (3 inondations successives, plusieurs dizaines de morts);

- 15 septembre 1741 (plusieurs morts, crue surnommée «le déluge »);

- 4 octobre 1768 (plusieurs morts);

- 2 octobre 1795 (un mort);

- 29 septembre 1815 ( 5 morts);

- Septembre et octobre 1834 ;

- 20 septembre 1846 (2 morts);

- 27-28 septembre, 8-9 octobre, 16-17 octobre 1907 ;

- 8 octobre 1933;

- 30 septembre et 4 octobre 1958 ( 36 morts).
Les riverains du Vidourle disent que les « vldourlades $\gg$ se produisent tous les vingt-cinq ans. Cette périodicité est sensiblement exacte pour les trois dernières grandes crues (1907, 1933, 1958). Elle est moins exacte auparavant. Mais il semble bien que la fréquence de ces grandes crues soit voisine de quatre par siècle.

On observera que ces grandes crues se produisent toujours en septembre et octobre.

Si l'on ne se limite plus à ces crues catastrophiques et si l'on fait un relevé des crues d'une certaine importance, leur répartition suivant les mois est la suivante :

$1^{\circ}$ Sur la Cèze, depuis 1892 , crues ayant atteint ou dépassé 7 mètres :

\begin{tabular}{|l|c|c|c|c|c|c|c|c|c|c|c|c|}
\hline $\mathrm{J}$ & $\mathrm{F}$ & $\mathrm{M}$ & $\mathrm{A}$ & $\mathrm{M}$ & $\mathrm{J}$ & $\mathrm{J}$ & $\mathrm{A}$ & $\mathrm{S}$ & $\mathrm{O}$ & $\mathrm{N}$ & $\mathrm{D}$ & Total \\
\hline 5 & & $\mathbf{1}$ & & $\mathbf{1}$ & $\mathbf{1}$ & $\mathrm{1}$ & & 4 & $\mathbf{9}$ & $\mathbf{1 0}$ & $\mathbf{2}$ & 34 \\
\hline
\end{tabular}

2 "Sur les Gardons, depuis 1897, crues ayant atteint on dépassé 5 mètres :

\begin{tabular}{|r|r|r|r|r|r|r|r|r|r|r|r|r|}
\hline $\mathrm{J}$ & $\mathrm{F}$ & $\mathrm{M}$ & $\mathrm{A}$ & $\mathrm{M}$ & $\mathrm{J}$ & $\mathrm{J}$ & $\mathrm{A}$ & $\mathrm{S}$ & $\mathrm{O}$ & $\mathrm{N}$ & $\mathrm{D}$ & Total \\
\hline 3 & 1 & & & & $\mathbf{1}$ & & & 4 & 6 & 1 & 2 & 18 \\
\hline
\end{tabular}


3" Sur le Vidourle, depuis 1900 , crues ayant atteint ou dépassé 4 mètres:

\begin{tabular}{|l|l|l|l|l|l|l|l|l|l|l|l|l|}
\hline$J$ & F & M & A & M & J & J & A & S & O & N & D & Total \\
\hline $\mathbf{1}$ & & & 2 & & 3 & & & 7 & 12 & 4 & 4 & 33 \\
\hline
\end{tabular}

On constate que 7 crues sur 8 se sont prodtiites pendant les mois de septembre inclus à janvier inclus. Encore convient-il d'observer que les crues qui se sont produites en dehors de la période septembre-janvier ne sont pas parmi les plus importantes.

On constate également que les crues se reproduisent souvent à quelques jours d'intervalle. On peut ainsi citer parmi les crues importantes:

\section{Sur la Cèze :}

Crues des 29 septembre et 3 octobre 1900; Crues des 9 octobre et 16 octobre 1907;

Crues des 27 septembre et 9 octobre 1933 :

Crues des 30 septembre et 4 octobre 1958.

\section{Sur les Gardons :}

Crues des 27 septembre, 9 octobre et 16 octobre 1907;

Crues des 27 septembre ot 9 octobre 1933;

Crues des 6 janvier et 31 janvier 1955 ;

Crues des 30 septembre et 4 octobre 1958.

\section{Sur le Vidourle:}

Crues des 27 septembre et 16 octobre 1907.

Les circonstances météorologiques qui provoquent les crues des rivières cévenoles se reproduisent souvent plusieurs fois à la mème époque de l'année.

$$
\text { : }
$$

\section{Conditions météorologiques}

Le tableau ci-dessous indique la vitesse du vent à différentes altitudes à la station de radiosondages de Nìmes-Courbessac.

\section{STATION DE RADIOSONDAGES DE NIMES-COURBESSAC \\ Direction et intensité du vent à différentes altitudes}

\begin{tabular}{|c|c|c|c|c|c|c|c|c|c|c|}
\hline \multirow{2}{*}{ Altitudes } & \multicolumn{2}{|c|}{29 septembre } & \multicolumn{2}{|c|}{30 septembre } & \multicolumn{2}{|c|}{3 octobre } & \multicolumn{2}{|c|}{4 octobre } & 5 octobre & \multirow{2}{*}{ OBSERVATIONS } \\
\hline & $0 \mathrm{~h}$ & $12 \mathrm{~h}$ & $0 \mathrm{~h}$ & $12 \mathrm{~h}$ & $0 \mathrm{~h}$ & $12 \mathrm{~h}$ & $0 \mathrm{~h}$ & $12 \mathrm{~h}$ & $12 \mathrm{~h}$ & \\
\hline au sol: & calme & $\begin{array}{l}13-07 \\
14-12\end{array}$ & $\begin{array}{l}09-09 \\
14-18\end{array}$ & $16-15$ & $\begin{array}{l}29-03 \\
32-05\end{array}$ & $\begin{array}{c}09-03 \\
14-06\end{array}$ & $\begin{array}{r}16-06 \\
15-15\end{array}$ & $\begin{array}{l}36-06 \\
36-09\end{array}$ & $\begin{array}{c}\text { Variable } \\
\text { faible } \\
21-03\end{array} \mid$ & $\begin{array}{l}\text { Ces renseignements } \\
\text { sont donnés suivant le } \\
\text { code ci-après: }\end{array}$ \\
\hline $500 \mathrm{~m}$ & $17-08$ & $14-12$ & $14-18$ & & $32-00$ & $14-00$ & $10-10$ & $30-0.9$ & $21-03$ & $1^{\prime}$ Les deux premiers \\
\hline $1000 \mathrm{~m}$ & $17-09$ & $16-14$ & $16-24$ & & $32-10$ & $16-09$ & $17-12$ & $12-06$ & $21-03$ & $\begin{array}{l}\text { chiffres indiquent la } \\
\text { direction du vent en }\end{array}$ \\
\hline $2000 \mathrm{~m}$ & $19-05$ & $19-14$ & $19-20$ & & $28-12$ & $20-14$ & $18-19$ & $14-12$ & $22-06$ & $\begin{array}{l}\text { rose de } 36 \\
\text { Nord : } 36 \text { ou } 0\end{array}$ \\
\hline $3000 \mathrm{~m}$ & $25-04$ & $20-10$ & $21-22$ & & $29-17$ & $21-11$ & $21-17$ & $17-17$ & $20-03$ & $\begin{array}{l}\text { Est : } 09 \\
\text { Sud : } 18\end{array}$ \\
\hline $4000 \mathrm{~m}$ & $26-06$ & $23-13$ & $21-19$ & $21-24$ & $29-18$ & $23-11$ & $21-20$ & $16-25$ & $23-12$ & Ouest : 27. \\
\hline $5000 \mathrm{~m}$ & $26-11$ & $24-10$ & $23-13$ & $22-29$ & $29-27$ & $23-16$ & $22-23$ & $19-26$ & $22-14$ & $2^{\circ}$ Les deux derniers \\
\hline $6000 \mathrm{~m}$ & $27-11$ & $25-12$ & $25-13$ & $22-17$ & $28-26$ & $23-20$ & $22-27$ & $23-16$ & $23-17$ & vitesse du vent en \\
\hline $7000 \mathrm{~m}$ & $27-09$ & $24-07$ & $24-13$ & $22-27$ & $28-30$ & $25-23$ & $22-24$ & $22-19$ & $23-21$ & \\
\hline $8000 \mathrm{~m}$ & $27-12$ & $25-06$ & $22-13$ & $22-34$ & & $25-26$ & $21-29$ & $22-19$ & $24-18$ & \\
\hline $9000 \mathrm{~m}$ & $27-12$ & $28-07$ & $23-18$ & $22-34$ & & $26-24$ & $22-26$ & $21-31$ & $24-22$ & \\
\hline $10000 \mathrm{~m}$ & $29-12$ & $31-05$ & $21-16$ & $22-22$ & & $25-31$ & $25-29$ & $21-29$ & $24-25$ & \\
\hline
\end{tabular}


Le deuxième tableau ci-dessous donne les directions et les vitesses du vent (en mètres par seconde) au mont Aigoual (altitude $1567 \mathrm{~m}$ ).

\begin{tabular}{|c|c|c|c|c|c|c|c|c|c|c|}
\hline Heures & \multicolumn{2}{|c|}{29 septembre } & \multicolumn{2}{|c|}{30 septembre } & \multicolumn{2}{|c|}{3 octobre } & \multicolumn{2}{|c|}{4 octobre } & \multicolumn{2}{|c|}{5 octobre } \\
\hline 0 heure.... & $S$ & 11 & S & 13 & $\mathrm{NNW}$ & 9 & SSE & 18 & $N W^{r}$ & 11 \\
\hline 3 heures. & $S$ & 12 & $S$ & 16 & $\mathrm{~W}$ & 2 & $S$ & 18 & NW & 13 \\
\hline 6 heures... & S & 12 & S & 17 & $S$ & 8 & $S$ & 15 & NW & 8 \\
\hline 9 heures... & $S$ & 12 & $S$ & 18 & SSE & 11 & $\mathrm{~W}$ & 11 & \multicolumn{2}{|c|}{ calme } \\
\hline 12 heures. & S & 13 & WNW & 13 & SSE & 12 & WNW & 7 & $S$ & 6 \\
\hline 15 heures... & $\mathrm{S}$ & 15 & WNW & 11 & $\mathrm{SSE}$ & 14 & NW & 7 & S & 4 \\
\hline 18 heures... & $S$ & 15 & NW & 8 & SSE & 16 & NW & 6 & NW & 9 \\
\hline 21 heures.... & $S$ & 14 & NNW & 7 & $S$ & 17 & NW & 8 & WNW & 13 \\
\hline
\end{tabular}

Ces tableaux montrent que le 29 septembre, veille de la crue du 30 septembre, le vent soufflait au sol du sud, et que le 3 octobre, veille de la crue du 4 octobre, le vent soufflait au sol du sud-sud-est.

En altitude, au-dessus de $3000 \mathrm{~m}$, le vent soufflait de l'ouest.

Il semble bien qu'un régime semblable de vents doit, d'une façon générale, précéder les crues importantes des rivieres cévenoles.$$
\text { * }
$$ \\ Chutes de pluie}

Nous indiquons ci-dessous, en allant de l'amont vers l'aval, les hauteurs de pluie tombées sur les stations pluviométriques de la Cèze et des Gardons les 29 et 30 septembre, du Vidourle les 3 et 4 octobre.

\section{Bassin de la Cèze}

Saint-Maurice-de-Ventalon :

- le 29 septembre, de $8 \mathrm{~h}$ à $22 \mathrm{~h}$...

- du 29 septembre, 22 h, au 30 septembre, 6 h $40 \ldots \ldots \ldots \ldots$. . $140 \mathrm{~mm}$

-. le 30 septembre:

$$
\begin{aligned}
& \text { de } 6 \mathrm{~h} 40 \text { à } 10 \mathrm{~h} \ldots \ldots \ldots \ldots . \ldots 5 \mathrm{~mm} \\
& \text { de } 10 \mathrm{~h} \text { à } 13 \mathrm{~h} \ldots \ldots \ldots \ldots \ldots . \ldots 9 \mathrm{~mm} \\
& \text { de } 13 \mathrm{~h} \text { à } 18 \mathrm{~h} 40 \ldots \ldots \ldots \ldots .65 \mathrm{~mm} \\
& 399 \mathrm{~mm}
\end{aligned}
$$

\section{Bességes :}

-..- du 29 septembre 7 h au 30 septembre $7 \mathrm{~h} \ldots \ldots \ldots \ldots \ldots \ldots \ldots$ 24 $\mathrm{mm}$

-... le 30 septembre:

$$
\text { de } 7 \mathrm{~h} \text { à } 13 \mathrm{~h} 30 \ldots \ldots \ldots .90 \quad 90 \mathrm{~mm}
$$

de 13 h 30 à $15 \mathrm{~h} 40 \ldots \ldots . \quad 86 \mathrm{~mm}$

- du 30 septembre $15 \mathrm{~h} 40$ au $1^{\mathrm{or}}$ octobre $7 \mathrm{~h} \ldots \ldots \ldots \ldots \ldots \ldots .67,6 \mathrm{~mm}$

\section{Saint-Ambroix :}

$$
267,6 \mathrm{~mm}
$$

- - du 30 septembre 7 h au $1^{\text {*r }}$ octobre 7 h. . . . . . . . . . . . . . . $185 \mathrm{~mm}$

\section{Bassin des Gardons}

a) Gardon D'AlÉs :

Collet-de-Déze :

- du 29 septembre 12 h au 30 septembre $7 \mathrm{~h} \ldots \ldots \ldots \ldots \ldots \ldots \ldots \ldots . . .173 \mathrm{~mm}$

- $-\mathrm{du} 30$ septembre $7 \mathrm{~h}$ au $1^{* \mathrm{r}}$ octobre $7 \mathrm{~h} \ldots \ldots \ldots \ldots \ldots \ldots \ldots$

$351 \mathrm{~mm}$

\section{La Grand-Combe:}

- le 30 septembre :

- de $0 \mathrm{~h}$ à $7 \mathrm{~h} \ldots \ldots . \ldots . . .30 \mathrm{~mm}$

$\ldots$ de $7 \mathrm{~h}$ à $12 \mathrm{~h} \ldots \ldots . \ldots 40 \mathrm{~mm}$

- de $12 \mathrm{~h}$ à $17 \mathrm{~h} \ldots \ldots \ldots . . .192 \mathrm{~mm}$

- de $17 \mathrm{~h}$ à $24 \mathrm{~h} \ldots \ldots . . . .24 \mathrm{~mm}$

$286 \mathrm{~mm}$ 
Intensité maxima de la pluie entre $12 \mathrm{~h}$ et $17 \mathrm{~h}$.

$$
\text { Alès : }
$$

- le 30 septembre :

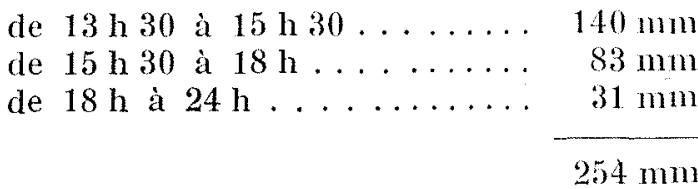

Intensité maxima de la pluie entre $13 \mathrm{~h} 30$ el $15 \mathrm{~h} 30$.

- le 4 octobre:

$$
\begin{aligned}
& \text { de } 8 \mathrm{~h} 30 \text { à } 10 \mathrm{~h} \ldots \ldots \ldots \ldots . .121 \mathrm{~mm} \\
& \text { de } 10 \mathrm{~h} \text { à } 12 \mathrm{~h} \ldots \ldots \ldots \ldots \ldots \ldots . . . . . .69 \mathrm{~mm} \\
& 160 \mathrm{~mm}
\end{aligned}
$$

Intensité maxima de la pluie le 4 octobre entre $8 \mathrm{~h} 30$ et $10 \mathrm{~h}$ avec une chute de $70,2 \mathrm{~mm}$ en trois quarts d'heure, soit 93,6 par heure.

\section{b) Gardon de Mialet :}

Burre des Cévennes:

-.. du 29 septembre 7 h au 30 septembre 7 h . . . . . . . . . . . du 30 septembre $7 \mathrm{~h}$ au 30 septembre $12 h \ldots \ldots \ldots \ldots$

$183 \mathrm{~mm}$

Saint-Etienne-Vallée française :

- du 29 septembre $8 \mathrm{~h}$ au 30 septembre $8 \mathrm{~h} \ldots \ldots \ldots \ldots \ldots$

- du 30 septembre $8 \mathrm{~h}$ au $1^{\text {er }}$ octobre $8 \mathrm{~h} \ldots \ldots \ldots \ldots \ldots \ldots \ldots$

$243 \mathrm{~mm}$

$429 \mathrm{~mm}$

c) Gardon de Saint-Jean.

Saint-André-de-Valborgne :

- du 29 septembre $7 \mathrm{~h}$ au 30 septembre $7 \mathrm{~h} \ldots \ldots \ldots \ldots \ldots \ldots \ldots$. . . $150 \ldots$

- le 30 septembre :

de $7 \mathrm{~h}$ à $10 \mathrm{~h} 30 \mathrm{mn} \ldots \ldots \ldots .62 \mathrm{~mm}$ de $10 \mathrm{~h} 30$ à $16 \mathrm{~h} 30 \mathrm{mn} \ldots \ldots .132 \mathrm{~mm}$

$344 \mathrm{~mm}$

\section{Saint-Jean-du-Gard :}

- du 29 septembre $8 \mathrm{~h}$ au 30 septembre $8 \mathrm{~h} \ldots \ldots \ldots \ldots \ldots$ 118,6 mm

- le 30 septembre:

de $8 \mathrm{~h}$ à $12 \mathrm{~h} \ldots \ldots \ldots \ldots \ldots . \ldots 107 \mathrm{~mm}$

de $12 \mathrm{~h}$ a $17 \mathrm{~h} \ldots \ldots \ldots \ldots \ldots . \ldots 161 \mathrm{~mm}$

de $17 \mathrm{~h}$ à $20 \mathrm{~h} \ldots \ldots \ldots \ldots \ldots .11,1 \mathrm{~mm}$

$397,7 \mathrm{~mm}$

d) Salindringue.

Lasalle:

_- Ie 30 septembre de $13 \mathrm{~h}$ a $17 \mathrm{~h} \ldots \ldots 105 \mathrm{~mm}$

e) Les Gardons réunis.

Uzès :

-_. le 30 septembre de $18 \mathrm{~h} \mathrm{à} 21 \mathrm{~h}$. . $80 \mathrm{~mm}$

- du 30 septembre $21 \mathrm{~h}$ au $1^{\text {er }}$ octobre $7 \mathrm{~h} \ldots \ldots \ldots \ldots \ldots \ldots .20,7 \mathrm{~mm}$

$100,7 \mathrm{~mm}$

\section{Bassin du Virdoule}

Saint-Hippolyte-du-Fort :

- le 4 octobre, de $5 \mathrm{~h}$ à $9 \mathrm{~h} \ldots \ldots \ldots .82 \mathrm{~mm}$

Quissac:

- du 3 octobre 7 h au 4 octobre 7 h

(en 4 heures).. $230 \mathrm{~mm}$

De ces relevés, il apparaît que les précipitations se sont déplacées de l'amont vers l'aval des vallées. Cette circonstance est notamment assez nette entre la Grand-Combe, où l'intensité. maxima a commencé vers $12 \mathrm{~h}$., et Alès, où la pluie a commencé à $13 \mathrm{~h} 30$. Ce décalage de 1 h $30 \mathrm{mn}$ est du même ordre de grandeur que le temps de propagation de la crue entre la la Grand-Combe et Alès. Cette circonstance, jointe à la forme des bassins supérieurs en éventail, favorise la concentration des débits à l'aval.

Notons également qu'on a relevé à Alès les intensités maxima suivantes :

- $140 \mathrm{~mm}$ en 120 minutes, soit une intensité de $1,18 \mathrm{~mm}$ par minute,

- $70,2 \mathrm{~mm}$ en 45 minutes, soit une intensité de $1,56 \mathrm{~mm}$ par minute.

La courbe que j'ai donnée pour Montpellier d'apres la formule Grisollet-Godard :

$$
\left(\mathrm{H} / \mathrm{T}=5,3^{\mathrm{T}-0,4}\right),
$$


à propos de la crue du Verdanson le 30 novembre 1955 à Montpellier, indique:

- pour une durée de 120 minutes, une intensitć de $0,8 \mathrm{~mm}$,

- pour une duréc de 45 minutes, une intensité de $1,15 \mathrm{~mm}$.

Il semble done que la formule Grisollet-Godard pour Montpellier ne s'applique pas dans les Cévennes, où la courbe reliant l'intensité maxima à la durée de l'averse paraît devoir se situer nettement au-dessus de la courbe de Montpellier, qui elle-même s'établissait nettement au-dessus de celle de Paris.

Ces deux intensités maxima à Alès donnent, sous la forme hyperbolique, la relation suivante :

$$
\frac{\mathrm{H}}{\mathrm{T}}=\frac{363}{\mathrm{~T}+188}
$$

\section{CONCLUSIONS}

Ces constatations et ces considérations montrent :

$1^{\circ}$ Que les chutes maxima de pluies se sont produites sur les hauts bassins cévenols, mais cependant à une certaine distance des crêtes;

$2^{\circ}$ Que ces violentes averses se sont déplacées de l'amont vers l'aval, circonstance qui, jointe à la forme en éventail des hauts bassins cévenols, a grandement favorisé la concentration des débits, et par conséquent la brutalité de la crue;

$3^{\circ}$ Que les renseignements météorologiques sur la direction et la vitesse des vents doivent permettre en général d'alerter les services intéressés quelques heures avant les crues de ces rivières.

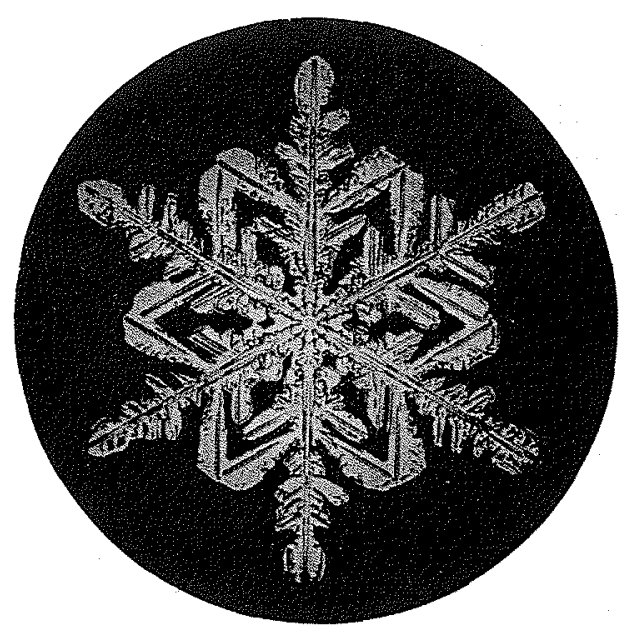

